\title{
A NOTE ON HUA'S INEQUALITY FOR COMPLEX NUMBERS
}

\author{
GOU-SHENG YANG AND BAE-KEWI HAN
}

\begin{abstract}
In this paper we give a general Hua's inequality involving a finite sequence of complex numbers.
\end{abstract}

\section{Introduction}

The following inequality of Lo-Keng Hua [1, P.149] is important in Number Theory.

Theorem 1. Let $\delta, \alpha>0$ and $x_{i} \in R(i=1, \cdots, n)$. Then

$$
\left(\delta-\sum_{i=1}^{n} x_{i}\right)^{2}+\alpha \sum_{i=1}^{n} x_{i}^{2} \geq \frac{\alpha}{n+\alpha} \delta^{2}
$$

with equality if and only if

$$
x_{i}=\frac{\delta}{n+\alpha}, i=1, \cdots, n
$$

The following generalization of Theorem 1 is due to Chung-Lie Wang [2]. See also [5].

Theorem 2. Let $\delta, \alpha>0$, and

$$
F_{n}(x)=\left(\delta-\sum_{i=1}^{n} x_{i}\right)^{p}+\alpha^{p-1}\left(\sum_{i=1}^{n} x^{p}\right) .
$$

Then for $p>1$, the inequality

$$
F_{n} \geq\left(\frac{\alpha}{n+\alpha}\right)^{p-1} \delta^{p}
$$

Received August 23, 1995.

1991 Mathematics Subject Classification. Primary 26D15.

Key words and phrases. Hua's inequality. 
holds for all nonnegative $x_{i} \in \mathbf{R}(i=1,2,3, \cdots, n)$ with $\sum_{i=1}^{n} x_{i} \leq \delta$.

The sign of enequality in (4) is reversed for $0<p<1$. In either case, the sign of equality holds if and only if (2) holds.

Recently, Dragomir [3] established an analogue inequality of (1) for complex numbers as follow.

Theorem 3. Let $\alpha>0$ and $\delta, z_{1}, z_{2}, \cdots, z_{n} \in \mathbf{C}$. Then the following inequality

$$
\left|\delta-\sum_{i=1}^{n} z_{i}\right|^{2}+\alpha \sum_{i=1}^{n}\left|z_{i}\right|^{2} \geq \frac{\alpha}{n+\alpha}|\delta|^{2}
$$

holds. The equality is valid in (5) if and only if

$$
z_{i}=\frac{\delta}{n+\alpha}, \quad i=1,2, \cdots, n .
$$

The main purpose of this note is to give a generalization of Theorem 3 .

\section{Gneralization of theorem 3}

Theorem 4. If $\alpha>0$, let $\delta, z_{1}, \cdots, z_{n} \in \mathbf{C}$ and

$$
F_{n}(z)=\left|\delta-\sum_{i=1}^{n} z_{i}\right|^{p}+\alpha^{p-1} \sum_{i=1}^{n}\left|z_{i}\right|^{p}
$$

The for $p>1$, we have

$$
F_{n}(z) \geq\left(\frac{\alpha}{n+\alpha}\right)^{p-1}|\delta|^{p}
$$

and the sign of equality holds in (16) if and only if

$$
z_{i}=\frac{\delta}{n+\alpha}, \quad i=1, \cdots, n .
$$

Proof. we need the following lemma.

Lemma 4.1. [4, P.26]: If $k>1$, and $k^{\prime}$ is the conjugate of $k$, then

$$
\left|\sum a b\right| \leq\left(\sum|a|^{k^{\prime}}\right)^{\frac{1}{k^{\prime}}}\left(\sum|b|^{k}\right)^{\frac{1}{k}}
$$

holds for any finite complex sequences $(a),(b)$. There is equality if and only if $\left(\left|a_{v}\right|^{k^{\prime}}\right)$ and $\left(\left|b_{v}\right|^{k}\right)$ are proportional and $\operatorname{arga}_{v} b_{v}$ is independent of $v$.

Now, let $\lambda=\delta-\sum_{i=1}^{n} z_{i}$. Then $z_{1}=\delta-\lambda-\sum_{i=2}^{n} z_{i}$. Applying (8) with $k=p>1$ to the following two sequences $(a),(b)$ :

$$
a_{1}=1, b_{1}=\lambda, a_{2}=\alpha^{\frac{1-p}{p}}, b_{2}=\left(\delta-\lambda-\sum_{i=2}^{n} z_{i}\right) \alpha^{\frac{p-1}{p}},
$$


and

$$
a_{i}=\alpha^{\frac{1-p}{p}}, b_{i}=z_{i-1} \alpha^{\frac{p-1}{p}} \text { for } 3 \leq i \leq n+1 .
$$

where $\sum_{i=1}^{n+1} a_{i} b_{i}=\delta$, we have

$$
\begin{aligned}
\mid \delta & \leq\left(1+n \alpha^{-1}\right)^{\frac{p-1}{p}}\left(|\lambda|^{p}+\left|\delta-\lambda-\sum_{i=2}^{n}\right|^{p} \alpha^{p-1}+\sum_{i=2}^{n}\left|z_{i}\right|^{p} \alpha^{p-1}\right)^{\frac{1}{p}} \\
& =\left(\frac{n+\alpha}{\alpha}\right)^{\frac{p-1}{p}}\left(F_{n}(z)\right)^{\frac{1}{p}},
\end{aligned}
$$

so that

$$
|\delta|^{p} \leq\left(\frac{n+\alpha}{\alpha}\right)^{p-1} F_{n}(z),
$$

which is equivalent to

$$
F_{n}(z) \geq\left(\frac{\alpha}{n+\alpha}\right)^{p-1}|\delta|^{P} .
$$

If the equality holds in (9), then

$$
\frac{|\lambda|}{1}=\frac{\left|\delta-\lambda-\sum_{i=2}^{n} z_{i}\right|}{\alpha^{-1}}=\frac{\left|z_{2}\right|}{\alpha^{-1}}=\cdots=\frac{\left|z_{n}\right|}{\alpha^{-1}} \text { with } z_{1}=\delta-\lambda-\sum_{i=2}^{n} z_{i},
$$

and

$$
\delta-\lambda-\sum_{i=2}^{n} z_{i}=t_{1} \lambda ; z_{i}=t_{i} \lambda, t_{i} \in \mathbf{R}_{+}, i=2 \cdots, n .
$$

It follows from (10) and (11) that

$$
z_{i}=\frac{\delta}{n+\alpha}, \quad i=1, \cdots, n
$$

Converserly, if

$$
z_{i}=\frac{\delta}{n+\alpha}, i=1,2, \cdots, n
$$

it is easily seen that

$$
F_{n}(z)=\left(\frac{\alpha}{n+\alpha}\right)^{p-1}|\delta|^{p} .
$$

This completes the proof.

Corollary. If $\alpha>0, \delta \in C$ and $\left(w_{i}\right)$ is a sequence of complex numbers such that $\sum w_{i}$ is convergent, then for $p>1$, we have

$$
\left|\delta-\sum w_{i}\right|^{p}+\alpha^{p-1}\left|\sum w_{i}\right|^{p} \geq\left(\frac{\alpha}{1+\alpha}\right)^{p-1}|\delta|^{p} .
$$

The equality holds in (12) if and only if

$$
\sum w_{i}=\frac{\delta}{1+\alpha}
$$


Proof. Let $z_{1}=\sum w_{i}$. Then applying Theorem 4 to the case $n=1$.

Remark. It is not necessarily true that the sign of the inequality in (7) is reversed for $0<p<1$.

For example, let

$$
p=\frac{1}{2}, \alpha=\delta=1, z_{1}=i \text { and } z_{3}=-1
$$

Then we have

$$
\begin{gathered}
F_{3}(z)=\left|1-\sum_{k=1}^{3} z_{k}\right|^{\frac{1}{2}}=3 \\
>2=\left(\frac{1}{3+1}\right)^{-\frac{1}{2}}
\end{gathered}
$$

\section{References}

[1] L. K. Hua, "Additive Theory of Prime Numbers(Translated by N.B. Ng) in Translations of Math. Monographs," Vol.13 Amer. Math. Soc. Providence, RI, 1965.

[2] C. L. Wang, "Lo-Keng Hua inequality and dynamic programming," J. Math. Anal., 166(1992), 345-350.

[3] S. S. Dragomir, "Hua's Inequality For Complex Numbers," Tamkang J. Math., Vol 26, No 3.(1995), 257-260.

[4] Hardy, Littlewood, and Polya, Inequalities, 2nd ed., Camb́ridge Univ. Press, Cambridge, 1964.

[5] C. E. M. Pearce, and J. E. Pečarič, "A remark on the Lo-keng Hua Inequality," J. Math. Anal., 188(1994), 700-702.

Department of Mathematics, Tamkang University, Tamsui, Taiwan, 25137. 